\title{
High resolution in situ magneto-optic Kerr effect and scanning tunneling microscopy setup with all optical components in UHV
}

\author{
A. Lehnert, P. Buluschek, N. Weiss, J. Giesecke, M. Treier, S. Rusponi, and H. Brune \\ Institute of the Physics of Nanostructures, Ecole Polytechnique Fédérale de Lausanne (EPFL), \\ Station 3, CH-1015 Lausanne, Switzerland
}

(Received 3 November 2008; accepted 10 January 2009; published online 9 February 2009)

\begin{abstract}
A surface magneto-optic Kerr effect (MOKE) setup fully integrated in an ultrahigh vacuum chamber is presented. The system has been designed to combine in situ MOKE and scanning tunneling microscopy. Magnetic fields up to $0.3 \mathrm{~T}$ can be applied at any angle in the transverse plane allowing the study of in-plane and out-of-plane magnetization. The setup performance is demonstrated for a continuous film of 0.9 monolayers $(\mathrm{ML}) \mathrm{Co} / \mathrm{Rh}(111)$ with in-plane easy axis and for a superlattice of nanometric double layer $\mathrm{Co}$ islands on $\mathrm{Au}(11,12,12)$ with out-of-plane easy axis. For $\mathrm{Co} /$ $\mathrm{Au}(11,12,12)$ we demonstrate that the magnetic anisotropy energies deduced from thermally induced magnetization reversal and from applying a torque onto the magnetization by turning the field are the same. For the presented setup we establish a coverage detection limit of 0.5 ML for transverse and 0.1 ML for polar MOKE. For island superlattices with the density of $\mathrm{Co} /$ $\mathrm{Au}(11,12,12)$, the latter limit corresponds to islands composed of about 50 atoms. The detection limit can be further reduced when optimizing the MOKE setup for either one of the two Kerr configurations. (C) 2009 American Institute of Physics. [DOI: 10.1063/1.3077148]
\end{abstract}

\section{INTRODUCTION}

Magnetism of low-dimensional systems differs substantially from the one of bulk matter and thereby attracts fundamental interest, which is further motivated by applications in ultrahigh density magnetic data storage. The field of lowdimensional magnetism stimulated the development of experimental techniques addressing the properties of magnetic nanostructures down to smallest sizes. Magneto-optic Kerr effect (MOKE) is readily used to probe magnetic multilayers, thin films, and more and more also nanostructures. The success of this technique is due to its relatively simple experimental implementation and its very good detection limit. For out-of-plane magnetized islands, the present limit has been in the range of 250 atoms. ${ }^{1}$ Here we present a setup improving the detection limit to 50 atoms, allowing application of magnetic torque with a vector field up to $0.3 \mathrm{~T}$, and offering vacuum conditions of $p_{\text {tot }}<1 \times 10^{-10}$ mbar, enabling the study of low-dimensional systems in their clean state. These features are achieved by mounting all optical components inside the ultrahigh vacuum (UHV) chamber. In addition, sample preparation, growth of nanostructures or films, as well as their characterization by means of scanning tunneling microscopy (STM) and MOKE take place with the sample at the same location offering full temperature control (60-1800 K).

Recent studies of small Co islands on $\mathrm{Pt}(111)$ have shown, by establishing a correlation between magnetism and morphology, that low coordinated atoms have significantly enhanced magnetocrystalline anisotropy energies (MAE). $\mathrm{X}$-ray magnetic circular dichroism revealed that isolated atoms have up to 200 times the bulk anisotropy ${ }^{2}$ and MOKE results showed that the magnetic anisotropy of large islands is determined by the perimeter atoms. ${ }^{3}$ Together with the fact that low coordinated atoms are highly reactive, ${ }^{4}$ this results in the magnetic properties being extremely sensitive to minor amounts of gas. Exposures to 0.05 Langmuir $(1 \mathrm{~L}$ $=10^{-6}$ mbar s) of $\mathrm{O}_{2}$ cause a $20 \%$ decrease in the blocking temperature $T_{b}$ of Co islands on $\mathrm{Pt}(111),{ }^{5}$ and $0.2 \mathrm{~L} \mathrm{O}_{2}$ the sudden appearance of out-of-plane remanence for $\mathrm{Fe}$ nanostripes on W(110), which have in-plane remanence in the clean state. ${ }^{6}$ This motivates the aim for good vacuum conditions. Precise temperature control is required first by the reproducibility of the growth morphology, necessitating to know the absolute temperature to within $1 \mathrm{~K}$, and second by mechanical stability during the acquisition of hysteresis loops, as well as during variable-temperature STM measurements, both requiring the relative temperature to be kept constant within $0.1 \mathrm{~K}$.

In conventional Kerr setups, the optics is mounted outside the UHV chamber and the probing laser light has to pass twice a UHV window. ${ }^{7}$ This gives rise to the Faraday effect caused by the magnetic stray field at the window location and produces birefringence. Both result in a residual signal, which is not sufficiently reproducible to be subtracted from the data, especially if one intends to probe small island sizes and low coverages. These effects are avoided in our setup by mounting all optical components into UHV thus improving the detection limit.

The magnetic field at the sample location can either be generated by placing the coils inside the UHV chamber close to the sample 7,8 or by mounting them outside the chamber. $^{9-11}$ Mounting the coils outside avoids outgassing of the coils when a current is passed through. For this reason we decided to mount the coils outside. In order to be able to address in- and out-of-plane magnetization, as well as to apply a magnetic torque, we chose a four pole electromagnet, ${ }^{11}$ 
(a)

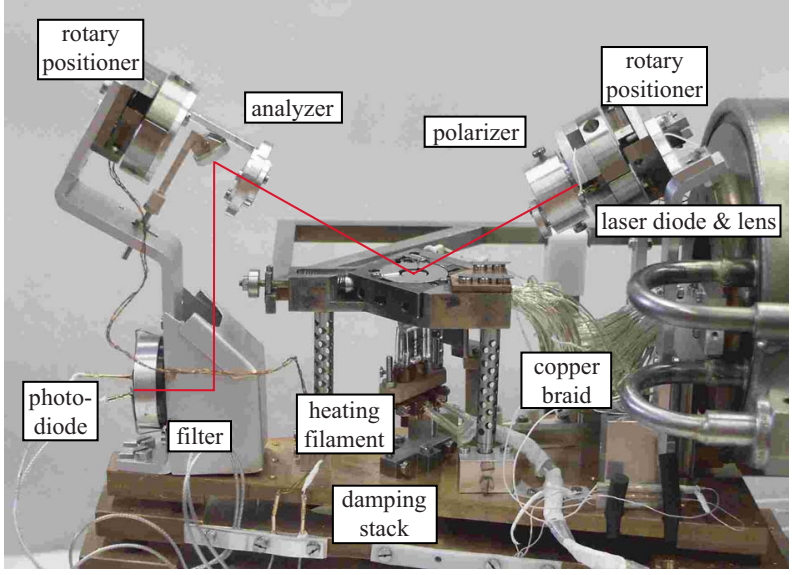

(b)

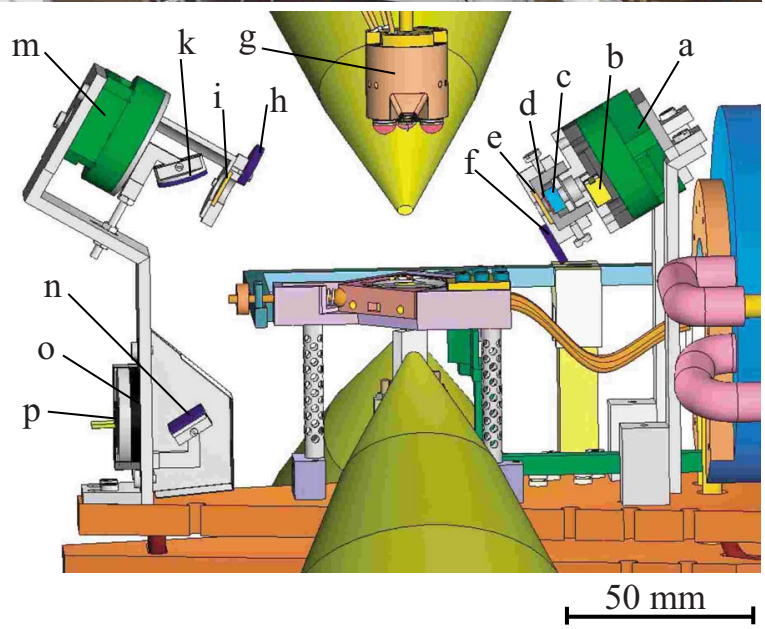

FIG. 1. (Color online) (a) Photograph of the MOKE setup with all optical components in UHV. The red line indicates the laser beam. All parts are mounted on a stack of copper plates separated by viton spacers for vibrational damping. (b) Technical drawing. Only three of the four poles (yellow cones) generating the magnetic field are shown. The STM is indicated in its rest position. It is lowered onto the sample for STM imaging. (a) Rotary positioner, (b) laser diode, (c) lens, (d) aperture, (e) polarizer, (f) shutter, (g) STM, (h) shutter, (i) analyzer, (k) mirror, (m) rotary positioner, (n) mirror, (o) filter, and (p) photodiode.

allowing measurements with the magnetic field vector pointing in any direction in the plane perpendicular to the one of the incident and reflected light. We chose to use the polartransverse Kerr geometry instead of the more common polarlongitudinal one. This choice is due to the fact that the polartransverse geometry lends itself better for the combination of in situ STM and Kerr measurements with a four pole magnet.

Overall this gives rise to a sensitivity better than 0.1 monolayers (ML) in polar and 0.5 ML in transverse geometry allowing the investigation of the magnetic properties of small clusters down to a few tens of atoms.

\section{EXPERIMENTAL SETUP}

A photograph of the sample stage with the MOKE optics is shown in Fig. 1(a). It is integrated into a UHV chamber, which has been designed to prepare and characterize the sample in the very same position. In addition, samples can be transferred to a manipulator for Auger electron spectroscopy, to a sample storage, and in and out via a fast entry lock. The sample is mounted as a sandwich in between sapphire rings on a molybdenum sample holder inserted in the sample stage. One wire for the sample potential and a $C$-type thermocouple are directly attached to the sample. Spring loaded contacts are mounted for these three wires onto the sample stage. The sample stage is connected with a silver plated copper braid to a liquid helium flux cryostat. A minimum sample temperature of $T=60 \mathrm{~K}$ can be reached during normal operation allowing sample exchange. This value may be lowered to $T \simeq 40 \mathrm{~K}$ when screwing the sample holder tightly to the sample stage, thus improving the thermal contact. The sample can be heated up to $T=2000 \mathrm{~K}$ via radiation and electron bombardment using a filament from a $50 \mathrm{~W}$ halogen light bulb placed close to the sample backside. The UHV chamber is further equipped with standard tools for surface preparation and characterization, such as an ion gun and atomic beam evaporators pointing to the sample stage. A home-built beetle type STM allows the characterization of the sample morphology. ${ }^{12}$

The technical drawing shown in Fig. 1(b) displays the components of the MOKE. The light source is a laser diode (Axcel Photonics, CL-785-0050-S90) emitting at $782 \mathrm{~nm}$. The laser is operated with a constant forward current of $I_{\text {laser }}=50 \mathrm{~mA}$ corresponding to an output power of $11 \mathrm{~mW}$ in air. In vacuum the laser cannot thermalize by convection anymore and therefore a good thermal anchoring of the laser chip was ensured by mounting it on a Au plated $\mathrm{Cu}$ block (Axcel Photonics). With this solution the threshold current increased by only $5 \%$ and the output power decreased by $30 \%$ at $I_{\text {laser }}=50 \mathrm{~mA}$. We note that standard laser diodes are usually mounted onto a base plate containing magnetic elements, which is not acceptable for the present setup.

The emitted beam is collimated by a plano-convex sapphire lens (Oriel, 74303) and passes an aperture creating a parallel beam with a diameter of about $1 \mathrm{~mm}$ along the entire optical path. The light is linearly polarized by two stacked dichroic sheets (codixx, VIS $700 \mathrm{BC} 4$ ), each of them having an extinction ratio of 1:80 000. They will be called polarizer in the following. Ideally, prism polarizers should be used having an extinction ratio better than 1:100 000. However, these prisms are held together by a refractive index matching glue, which is neither UHV compatible nor bakeable. Laser diode, lens, aperture, and polarizer are rigidly mounted on a piezodriven rotary positioner with $360^{\circ}$ travel and a minimum step size of $0.001^{\circ}$ (attocube, ANR100). This allows continuous adjustment of the polarization direction from $s$ to $p$, as well as opening/closing a front-end shutter. The shutter protects the sheet polarizers against $\mathrm{Ar}^{+}$ion bombardment and metallization when the sample is sputtered or metallic films are deposited, respectively, both having the long term drawback of deteriorating the performance of the polarizer.

The laser beam is reflected by the sample and hits another pair of dichroic sheets, which we call analyzer. The analyzer is mounted on an identical rotary positioner as the polarizer allowing to freely adjust the relative angle and to open/close a protective shutter during sample preparation, as well. Two high quality mirrors (WZWOPTICAG, S18x12x2.5-M01 and SD10x2-M01) deflect the beam onto a photodiode (Hamamatsu, S1337-1010BQ) measuring the intensity. To retain parasitic light the photodiode is screened by a filter (Oriel, 780FS10-25) with its maximum transmission 
centered around $783 \pm 5 \mathrm{~nm}$. With this setup we obtain extinction ratios better than 1:40000 on single crystal metal samples, depending on the quality of the surface polishing. The extinction ratio is defined as the ratio between the minimum light intensity $I_{\min }$, measured by the photodetector when polarizer and analyzer are oriented perpendicular, and the maximum light intensity $I_{\max }$ when polarizer and analyzer are oriented parallel to each other. The definitions of $I_{\min }$ and $I_{\max }$ will be used further below.

The mechanical supports of both rotary positioners and the sample stage are fixed on top of the uppermost plate of a passive damping stack reducing mechanical vibrations for Kerr and STM measurements. The incident angle $\theta$ with respect to the surface normal can be adjusted to $60^{\circ} \pm 10^{\circ}$ by ex situ positioning of the laser with respect to the sample. The laser beam is in the $y-z$ plane of the coordinate system chosen in Fig. 2.

Special care was taken to use only nonmagnetic materials for all parts seen in Fig. 1(a). Mechanical components were made of aluminum and copper while titanium was used for screws and bolts. This is of prime importance in order to avoid artifacts in the measured intensity caused when single components move with respect to each other driven by the applied magnetic field. For simplicity of the UHV Kerr setup and for keeping mechanical noise for STM at a minimum, we avoided the use of a photoelastic modulator. Based on our experience, all components are UHV compatible and bakeable at $120^{\circ} \mathrm{C}$ even though for some optical parts only temperatures up to $80{ }^{\circ} \mathrm{C}$ are guaranteed by the manufacturer. We routinely achieved a base pressure $<1 \times 10^{-10}$ mbar.

Figure 2(a) shows the four pole electromagnet with the sample being located in the center (see Fig. 1). It creates a magnetic field at any angle $\varphi$ in the $x-z$ plane, $\varphi$ being the angle included in between the applied field vector $\mathbf{H}$ and the surface normal $z$. We used four water cooled coils (GMW Inc.) situated outside the UHV chamber as shown in Fig. 2. Each of them produces a field of $120 \mathrm{mT}$ at a maximum applied current of $40 \mathrm{~A}$. Tapered soft iron cores (yellow cones in Figs. 1 and 2) are welded into bored CF 100 flanges, which bring the magnetic field to the sample. The cores are continued outside the vacuum chamber by cylinders (light blue in Fig. 2), which can by removed for disassembly. The soft iron yoke (red parts in Fig. 2) closes the magnetic field lines, reducing stray fields. Poles, cylinders, and yoke are made of ARMCO iron. In order to prevent oxidation, the parts exposed to air are painted while the vacuum side of the poles was coated with a $12 \mu \mathrm{m}$ thick nickel-phosphor layer.

The shape, dimensions, and arrangement of the poles were optimized by finite element calculations to obtain maximum field homogeneity and amplitude at the sample location while leaving enough room for the STM. The horizontal distance between the poles is $30 \mathrm{~mm}$ allowing to pass with the STM, having a diameter of $21 \mathrm{~mm}$. The maximum magnetic field in the sample region measured with a Hall sensor amounts to $B_{\max }=312 \mathrm{mT}$ along the $z$ or $x$ directions. The measured value is $10 \%$ larger than the one predicted from finite element calculations. For $\varphi=45^{\circ}$ the maximum field amounts to $222 \mathrm{mT}$. $B_{\max }$ changes by $2 \%$ when moving from
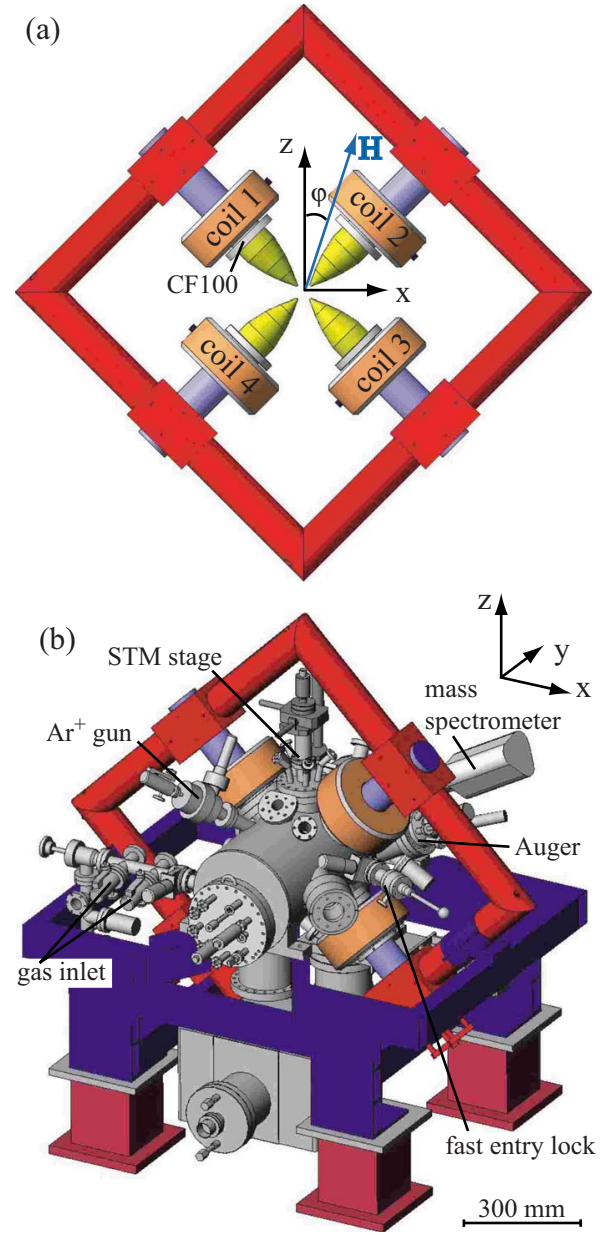

FIG. 2. (Color online) (a) Drawing of the four pole electromagnet. The poles (yellow) are welded into CF100 flanges and screwed on the UHV chamber. The poles having a diameter of $96 \mathrm{~mm}$ are continued by a cylinder (light blue) entering a square shaped yoke (red) surrounding the UHV chamber. Yoke, cylinders, and coils can easily be unmounted for chamber bakeout. The sample sits in the center of the four poles (see Fig. 1 for details). $\varphi$ is the angle between the applied field vector $\mathbf{H}$ and the surface normal z. (b) Drawing of the UHV chamber showing how the poles, coils, and yoke are mounted on the chamber. The positions of STM, Auger, $\mathrm{Ar}^{+}$ion gun, and fast entry lock are indicated.

the sample center $(x=0 \mathrm{~mm})$ to its edge $(x=4 \mathrm{~mm})$. In the region probed with the laser beam, having a spot diameter of $1 \mathrm{~mm}$, the field variation is less than $0.5 \%$. Thus, the magnetic field can be considered homogeneous within the area of the laser spot.

The soft iron poles have the intrinsic property of a remanent magnetization, which depends on the amplitude and sweep rate of the magnetic field. This remanence creates a residual field varying from 2 to $7 \mathrm{mT}$ for typical sweep rates ranging from 0.2 to $1 \mathrm{~T} / \mathrm{min}$ and for sweeps going up to the maximum field. The remanence has to be corrected for in the $M(H)$ curves.

\section{THEORETICAL BACKGROUND}

We opted for the plane of incidence perpendicular to the magnetic field leaving the largest room to fit the optical components and to adjust the incident angle $\theta$. $\theta$ has to be chosen carefully when in-plane and out-of-plane magnetized samples shall be measured at the same time, or when the 
Kerr geometry is to be optimized for either of the two, since the Kerr signal critically depends on it. We calculated the Kerr signal for polar and transverse geometry as a function of $\theta$ by applying the macroscopic formalism for magnetic multilayers. ${ }^{713}$ It has been shown that the macroscopic description of the magneto-optic effects breaks down in the mono- or submonolayer regime. ${ }^{14}$ Nevertheless, it correctly reproduces the global trends which is sufficient for the present purpose.

The Kerr effect manifests itself in the change of polarization (polar Kerr effect) or intensity (transverse Kerr effect) of polarized light when reflected from the surface. This phenomenon is summarized by the Fresnel coefficients linking the electric field amplitudes of the reflected wave $\left(E_{s}^{r}, E_{p}^{r}\right)$ to the ones of the incident wave $\left(E_{s}^{i}, E_{p}^{i}\right),{ }^{15}$

$$
\left(\begin{array}{c}
E_{s}^{r} \\
E_{p}^{r}
\end{array}\right)=\left(\begin{array}{ll}
r_{s s} & r_{s p} \\
r_{p s} & r_{p p}
\end{array}\right)\left(\begin{array}{c}
E_{s}^{i} \\
E_{p}^{i}
\end{array}\right) .
$$

We calculate the Fresnel coefficients for polar and transverse Kerr geometry for the systems which we investigated experimentally consisting of the three following media: vacuum, a thin Co film, and the substrate (Au or $\mathrm{Rh}$ ).

For this, we write the $4 \times 4$ medium boundary matrices $A$ and the $4 \times 4$ medium propagation matrix $D$ of the magnetic Co film of thickness $d$, with $A$ and $D$ being given in Ref. 7. Then we compute

$$
M=A_{\text {vacuum }}^{-1} A_{\text {film }} D_{\text {film }} A_{\text {film }}^{-1} A_{\text {substrate }} .
$$

The $4 \times 4$ matrix $M$ can be expressed by blocks of $2 \times 2$ submatrices.

$$
M=\left(\begin{array}{cc}
G & H \\
I & J
\end{array}\right),
$$

being related to the Fresnel coefficients by taking $I G^{-1}$.

We used the refractive index $n=1$ for vacuum and the following bulk refractive indices found in the literature at the laser wavelength $\lambda=782 \mathrm{~nm}: n_{\mathrm{Co}}=2.45+4.74 i,{ }^{16} n_{\mathrm{Au}}=0.16$ $+4.69 i,{ }^{17,18}$ and $n_{\mathrm{Rh}}=2.63+6.69 i{ }^{19}$ The Voigt constant for Co bulk at the given wavelength is $Q=0.04-0.02 i{ }^{20}$ Figure 3 shows the Kerr rotation and the Kerr asymmetry at a fixed film thickness of $d=0.2 \mathrm{~nm}$ as a function of the incident angle $\theta$ for a saturated sample. The Kerr rotation is given by $\operatorname{Re}\left(r_{s p} / r_{p p}\right)$ for an incident $p$-polarized wave and by $\operatorname{Re}\left(r_{p s} / r_{s s}\right)$ for an incident $s$-polarized wave. The Kerr asymmetry is defined as

$$
\frac{\left|r_{p p}(+M)\right|^{2}-\left|r_{p p}(-M)\right|^{2}}{\left|r_{p p}(+M)\right|^{2}+\left|r_{p p}(-M)\right|^{2}}
$$

for $p$-polarized light. Note that $s$-polarized light does not give rise to a transverse magneto-optic effect, i.e., the asymmetry is zero.

The polar Kerr rotation has a large and almost constant signal up to about $\theta=60^{\circ}$ diminishing rapidly for larger angles, whereas the transverse Kerr asymmetry has a clear maximum around $\theta=80^{\circ}$. Choosing an angle of incidence of $\sim 60^{\circ}$ results in a still very good polar Kerr signal, while being able to detect a reasonable transverse Kerr signal. The measurements presented in Sec. IV are carried out with $\theta=62^{\circ}$. However, the MOKE setup can be optimized for
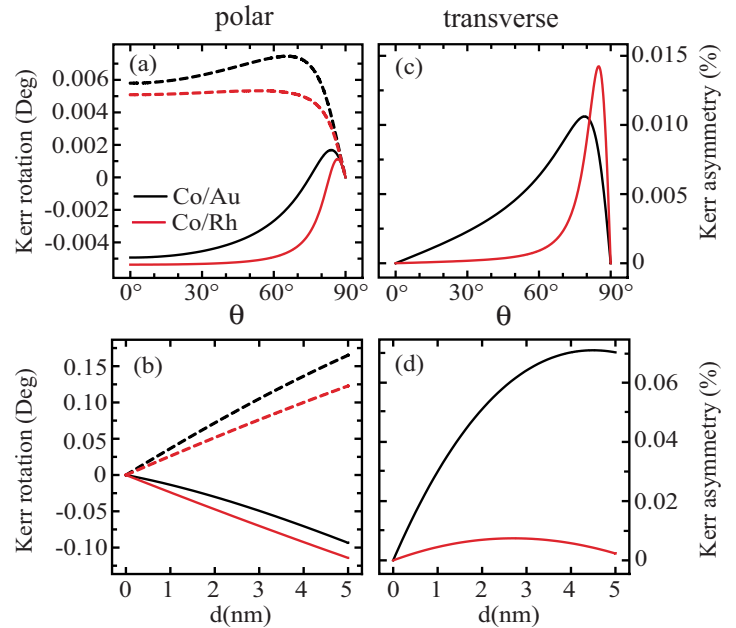

FIG. 3. (Color online) [(a) and (b)] Calculated Kerr rotation and [(c) and (d)] asymmetry as a function of the angle of incidence $\theta$ for [(a) and (c)] a film thickness of $d=0.2 \mathrm{~nm}$ and [(b) and (d)] as a function of film thickness at fixed angle of incidence $\theta=62^{\circ}$ for a Co film on Au (black lines) and $\mathrm{Rh}$ (red lines) at $\lambda=782 \mathrm{~nm}$ laser wavelength. [(a) and (b)] Polar Kerr: $\operatorname{Re}\left(r_{s p} / r_{p p}\right)$ is shown for $p$-polarized light (full line) and $\operatorname{Re}\left(r_{p s} / r_{s s}\right)$ for $s$-polarized light (dashed line). [(c) and (d)] Transverse Kerr: Kerr asymmetry for $p$-polarized light.

polar or transverse Kerr configuration by changing $\theta$ in order to improve the Kerr signal and therewith the detection limit for a given system.

\section{MEASUREMENTS}

The sensitivity of the MOKE setup was tested on two different systems: (A) a continuous Co film on $\mathrm{Rh}(111)$, having in-plane easy axis, and (B) an array of bilayer Co islands on $\mathrm{Au}(11,12,12)$ with out-of-plane easy axis. The surface of the $\mathrm{Rh}(111)$ single crystal was prepared by repeated cycles of $\mathrm{Ar}^{+}$ion sputtering $\left(1.3 \mathrm{keV}, 300 \mathrm{~K}, 3 \mu \mathrm{A} / \mathrm{cm}^{2}\right)$, annealing in oxygen $\left(3 \times 10^{-8} \mathrm{mbar}, 800 \mathrm{~K}\right)$, and flash annealing at $1500 \mathrm{~K}$. For $\mathrm{Au}(11,12,12)$ repeated cycles of $\mathrm{Ar}^{+}$ ion sputtering $\left(0.9 \mathrm{keV}, 300 \mathrm{~K}, 2 \mu \mathrm{A} / \mathrm{cm}^{2}\right)$ and annealing at $900 \mathrm{~K}$ were applied. Co was evaporated with an e-beam evaporator from a high purity rod $(99.995 \%)$ onto the sample with a flux of $0.26 \pm 0.02 \mathrm{ML} / \mathrm{min}$.

\section{A. $\mathrm{Co} / \mathrm{Rh}(111)$}

To determine the detection limit for transverse MOKE, we deposited 0.9 ML Co on $\mathrm{Rh}(111)$ at room temperature. Magnetization loops were taken of the as deposited film in the transverse and polar Kerr geometry. For the transverse configuration, 10 loops were recorded in order to obtain a better signal-to-noise ratio. The average and the standard error are displayed at each field in Fig. 4(a) evidencing a square loop with a sharp jump of the magnetization at the switching field. This shape is indicative of an in-plane easy magnetization axis. Assuming as a sensitivity criterium that the Kerr signal has to be at least as large as the noise level, we derive $0.5 \mathrm{ML}$ as the detection limit for the transverse geometry.

In polar geometry, the curve is more s-shaped and the magnetization does not reach saturation at the maximum available field [see Fig. 4(b)]. Here, we took three hysteresis 


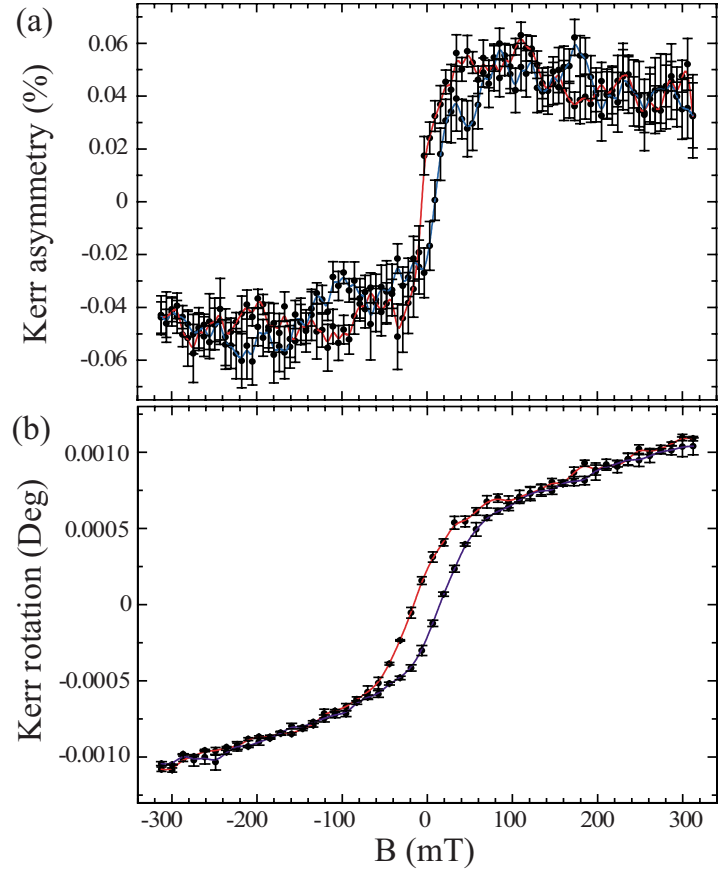

(c)

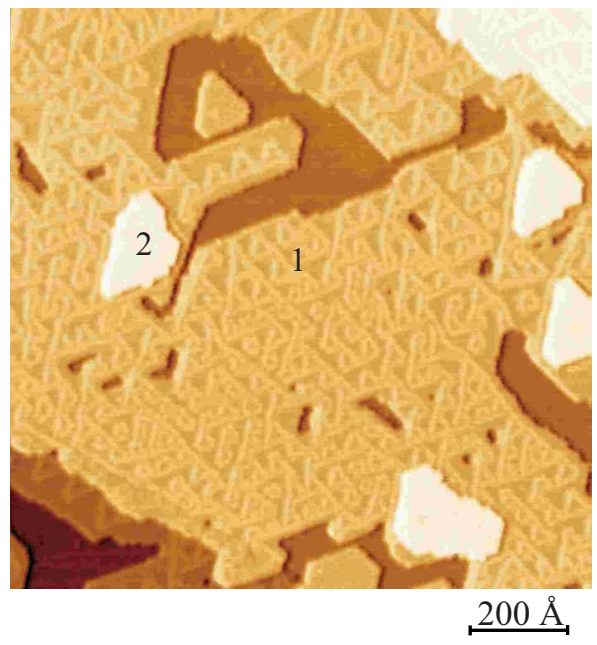

FIG. 4. (Color online) $0.9 \mathrm{ML} \mathrm{Co/Rh(111)} \mathrm{deposited} \mathrm{and} \mathrm{characterized} \mathrm{at}$ $T=300 \mathrm{~K}$. (a) transverse Kerr asymmetry using $p$-polarized light (average of ten hysteresis loops; forward sweep blue, backward sweep red). The error bars represent the standard error (standard deviation $/ \sqrt{N}$, where $N$ is the number of hysteresis loops). (b) Polar Kerr rotation using $p$-polarized light (average of three hysteresis loops; forward sweep blue, backward sweep red), (c) STM image of the deposited film $\left(V_{t}=-1.5 \mathrm{~V}, I_{t}=2.7 \mathrm{nA}\right.$, $T=300 \mathrm{~K})$. In order to increase the contrast, the image is represented as if the surface was illuminated from the left. The $\mathrm{Rh}(111)$ surface remains partially uncovered, most of it is covered by one atomic layer of Co (label 1) and a few islands grow in the second layer (label 2). The partial surface dislocations have an apparent height of $0.4 \AA$. The peak to peak noise of the STM is $\Delta z=0.05 \AA$. Note that the first and second Co layers are both reconstructed.

loops for averaging. For comparison with the calculations in Sec. III, we represented the Kerr rotation $\Phi$ for polar geometry. $\Phi$ is obtained from

$$
\Phi=\frac{\Delta I}{I_{0}(\delta)} \frac{\delta}{2}
$$

where $\Delta I$ is the measured intensity variation as a function of the applied field, $I_{0}(\delta)$ is the intensity in absence of an applied field, and $\delta$ is a small angle of the analyzer off total extinction. ${ }^{7} \quad \delta=0.5^{\circ}$ was estimated assuming $I_{0}(\delta)=I_{\min }$ $+I_{\max } \sin ^{2} \delta$ (for this sample we measured an extinction ratio of $\left.I_{\min } / I_{\max }=1: 47000\right)$. The polar Kerr measurement has a larger signal $\left(\Phi=0.001^{\circ}\right.$ corresponds to $\left.\Delta I / I_{0}(\delta)=0.4 \%\right)$ and consequently a much better signal-to-noise ratio compared to the transverse one, as we expect from the calculated curves. For an angle of incidence of $62^{\circ}$ and a $0.2 \mathrm{~nm}$ thick saturated Co film the calculated polar Kerr signal is almost ten times the transverse signal. From the signal-to-noise ratio in Fig. 4(b) we derive a detection limit of 0.10 ML for out-of-plane magnetized films.

An STM image of the Co film is shown in Fig. 4(c). Room temperature growth results in interconnected large Co patches, one atomic layer high, leaving about $15 \%$ of the substrate uncovered. 0.05 ML of Co grows in the second layer in form of small compact islands. Both Co layers show partial surface dislocations imaged as protrusions, separating fcc from hcp stacking areas. This way part of the stress resulting from the lattice mismatch between $\mathrm{Co}$ and $\mathrm{Rh}$ of $\left(a_{\mathrm{Co}}-a_{\mathrm{Rh}}\right) / a_{\mathrm{Rh}}=-6.6 \%$, where $a$ is the in plane nearest neighbor distance, is released.

\section{B. $\mathrm{Co} / \mathrm{Au}(11,12,12)$}

$\mathrm{Co} / \mathrm{Au}(11,12,12)$ forms an array of nanometric islands and therefore is an ideal model system for testing the performance of the UHV MOKE. ${ }^{1,21,22}$ The islands are monodomain magnets since for an island diameter of about $30 \AA$ a domain wall is energetically unfavorable. ${ }^{23,24}$ Further, they have a very narrow size distribution and their easy axis is out-of-plane.

$\mathrm{Au}(11,12,12)$ is a stable $\mathrm{Au}(111)$ vicinal surface with a terrace width of $58 \AA \AA^{21}$ The (111) oriented terraces exhibit a reconstruction relieving part of the tensile surface stress by the insertion of additional Au atoms into the topmost layer. $\mathrm{On} \mathrm{Au}(111), 23 \mathrm{Au}$ surface atoms per unit cell sit on 22 bulk lattice sites leading to fcc and hcp stacked areas. ${ }^{25}$ On the vicinal surface partial surface dislocations between these areas run perpendicular to the steps. Upon Co deposition, two atomic layer high Co islands nucleate at the crossing of a surface dislocation and the step edge, thus leading to an array of Co islands with a $58 \times 72 \AA^{2}$ periodicity and good long range order as shown in Fig. 5(d) ${ }^{21,22}$ To avoid coalescence, the sample was prepared by successively depositing $0.28 \mathrm{ML}$ Co at $T=130 \mathrm{~K}$ followed by annealing at $T=300 \mathrm{~K}$ until a total coverage of $1.1 \mathrm{ML}$ was reached. ${ }^{1}$ The island size distribution obtained from STM images has a Gaussian shape with a mean size of $\langle s\rangle=600$ atoms and a half width at half maximum of $27 \%$ of the mean size.

Our experimental setup allows to determine the island MAE by two independent methods, namely, by evaluating hysteresis loops acquired at different field angles $\varphi$ below the blocking temperature, and by measuring the temperature dependence of the zero-field susceptibility $\chi(T)$ $=d M(T) /\left.d H\right|_{H=0}$. We first focus on the first method. Figure 5(a) shows a hysteresis loop for $\varphi=0^{\circ}$ measured at $T$ $=75 \mathrm{~K}$ with a sweep rate of $1.0 \mathrm{~T} / \mathrm{min}$. Figure 5(b) represents the angular dependence of the magnetization at $B=222 \mathrm{mT}\left(M_{222}\right)$ and of the remanent magnetization $M_{R}$ 

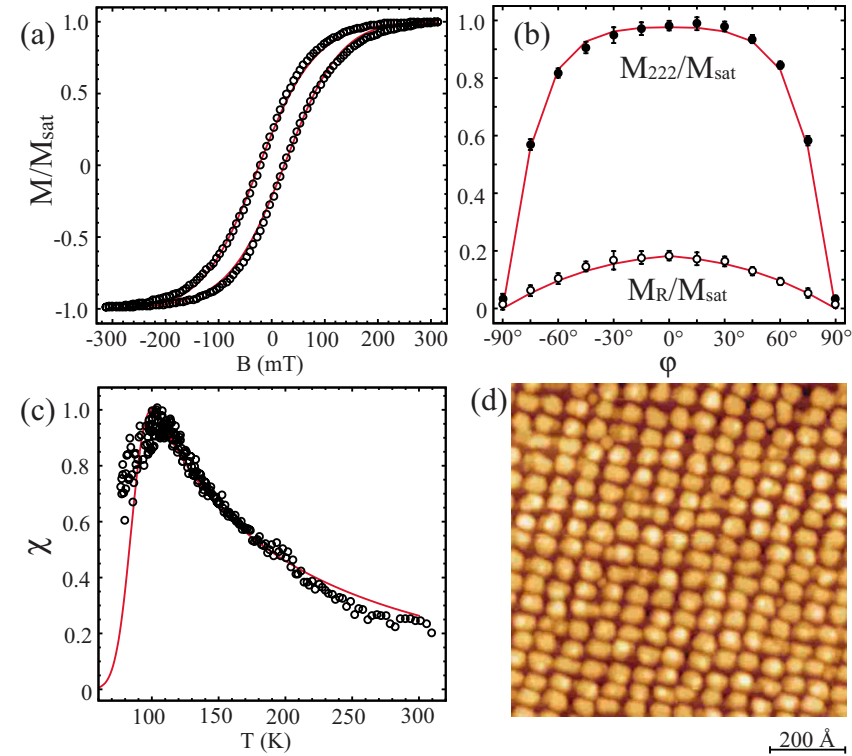

FIG. 5. (Color online) 1.1 ML Co/Au(11,12,12). (a) Hysteresis loop measured at $T=75 \mathrm{~K}$ in polar geometry (average of two hysteresis loops). The error bar is smaller than the symbol size and therefore not shown. Hysteresis loops were simulated assuming the island MAE to be proportional to the island perimeter length (solid line). (b) Magnetization at $B=222 \mathrm{mT}$ (full circles) and remnant magnetization (empty circles) measured at $T=75 \mathrm{~K}$. The solid curves represent $M_{222}$ and $M_{R}$ from simulated hysteresis curves. (c) $\chi(T)$ was measured by applying a triangular field sweep with a sweep rate of $13 \mathrm{~Hz}$ and $\pm 5 \mathrm{mT}$ amplitude (empty circles). The solid line represents the fit calculated assuming as in (a) and (b) island MAE to be proportional to the island perimeter length. (d) STM image of the nanoisland array $\left(V_{t}=-1.8 \mathrm{~V}, I_{t}=0.2 \mathrm{nA}, T=200 \mathrm{~K}\right)$.

taken from hysteresis loops recorded with a sweep rate of 1.0 $\mathrm{T} / \mathrm{min}$ and a maximum field of $B=222 \mathrm{mT}$ at $75 \mathrm{~K}$. The $M_{R} / M_{\text {sat }}$ versus $\varphi$ data clearly show that the easy axis is out-of-plane in agreement with earlier publications. ${ }^{1,22,26}$

The magnetization can be modeled by taking into account nonequilibrium effects of a thermally excited bistable system subjected to a time varying magnetic field. ${ }^{27}$ Any hysteresis loop for an ensemble of noninteracting monodomain particles with uniaxial anisotropy represents mainly the asymmetry in the number of particles pointing up $n_{\uparrow}$ and down $n_{\downarrow}$ changing over time with the applied field. The time evolution of $n_{\uparrow}$ can be expressed by the following master equation:

$$
\frac{d n_{\uparrow}}{d t}=-\kappa_{\uparrow \downarrow} n_{\uparrow}+\kappa_{\downarrow \uparrow} n_{\downarrow},
$$

where $\kappa_{\uparrow \downarrow}$ is the transition rate from $n_{\uparrow}$ to $n_{\downarrow}$ given by

$$
\kappa_{\uparrow \downarrow}=\nu_{0} e^{-E_{\uparrow \downarrow} \backslash k_{B} T} .
$$

$\nu_{0}$ is the attempt frequency, $E_{\uparrow \downarrow}$ is the barrier height, and $k_{B}$ is the Boltzmann constant. The energy landscape of a single Co island of size $s$, magnetic moment per atom $m$, and anisotropy energy $K$ is given by

$$
E=K \sin ^{2} \alpha-\operatorname{sm} H \cos (\alpha-\varphi),
$$

where $\alpha$ denotes the angle between $m$ and the easy axis, and $\varphi$ the angle of the magnetic field with respect to the easy axis, which is in this case the surface normal. The master Eq. (6) has been integrated numerically using $\nu_{0}=10^{10} \mathrm{~Hz}$ and $m=2.0 \mu_{B} /$ Co atom, ${ }^{1,3}$ while taking into account the island size and perimeter length distribution experimentally determined from STM images. Assuming the magnetic anisotropy energy to be proportional to the perimeter length $K=p K_{p}$, where $p$ is the number of perimeter atoms in the first and second layer, ${ }^{1,3}$ produces the best fit of the experimental curves shown in Figs. 5(a) and 5(b) with $K_{p}$ $=0.87 \pm 0.01 \mathrm{meV} /$ perimeter atom. This MAE value is compared with the one deduced from the second method using the temperature dependent susceptibility. $\chi(T)$, displayed in Fig. 5(c), was measured while cooling by applying a triangular field sweep with a sweep rate of $13 \mathrm{~Hz}$ and $\pm 5 \mathrm{mT}$ amplitude. The solid line is calculated using Eqs. (1) and (2) of Ref. 3 with $\nu_{0}=10^{10} \mathrm{~Hz}, m=2.0 \mu_{B} / \mathrm{Co}$ atom, $K_{p}$ $=0.95 \pm 0.01 \mathrm{meV} /$ perimeter atom, and the experimental island size and perimeter length distributions.

The fact that both methods give very similar anisotropy energies is showing that the barrier for thermal magnetization reversal is identical to the one obtained by tilting the magnetization vector away from the easy axis by a torque. Therefore the transition state in thermal magnetization reversal is monodomain for the presented system. The $K$ values compare very well to each other and with the ones found in earlier publications. ${ }^{1,22,28}$ We also note that the curve in Fig. 5(c) is entirely reversible; the same curve is obtained when heating. In addition the perfect agreement between fit and data for $T>T_{b}$ shows that the islands are noninteracting, since the fit is based on the superposition principle and interactions would give rise to a more shallow decay of $\chi \cdot{ }^{29}$ As a final comment we note that for 1.1 ML Co on Au we are far above the detection limit. Taking into account that for small coverages the Kerr signal is proportional to the thickness [see Fig. 3(b)] we estimate a detection limit for polar Kerr of about 0.1 ML. This corresponds to a mean island size of 50 atoms for the same island density as in the present experiment, provided that saturation magnetization is reached.

\section{ACKNOWLEDGMENTS}

We wish to thank the mechanical workshop of the Institute of the Physics of Nanostructures for the realization of the described experimental setup. Financial support from the Swiss National Science Foundation (SNSF Grant Nos. 21-58926.99, 2000-067218, 200020-104133, 200020109800, and 200020-112322) is gratefully acknowledged.

${ }^{1}$ N. Weiss, T. Cren, M. Epple, S. Rusponi, G. Baudot, S. Rohart, A. Tejeda, V. Repain, S. Rousset, P. Ohresser, F. Scheurer, P. Bencok, and H. Brune, Phys. Rev. Lett. 95, 157204 (2005).

${ }^{2}$ P. Gambardella, S. Rusponi, M. Veronese, S. S. Dhesi, C. Grazioli, A. Dallmeyer, I. Cabria, R. Zeller, P. H. Dederichs, K. Kern, C. Carbone, and H. Brune, Science 300, 1130 (2003).

${ }^{3}$ S. Rusponi, T. Cren, N. Weiss, M. Epple, P. Buluschek, L. Claude, and H. Brune, Nature Mat. 2, 546 (2003).

${ }^{4}$ S. Dahl, A. Logadottir, R. C. Egeberg, J. H. Larsen, I. Chorkendorff, E. Törnqvist, and J. K. Nørskov, Phys. Rev. Lett. 83, 1814 (1999).

${ }^{5}$ T. Cren, S. Rusponi, N. Weiss, M. Epple, and H. Brune, J. Phys. Chem. B 108, 14685 (2004).

${ }^{6}$ H. J. Elmers, J. Hauschild, and U. Gradmann, J. Magn. Magn. Mater. 198, 222 (1999).

${ }^{7}$ Z. Q. Qiu and S. D. Bader, Rev. Sci. Instrum. 71, 1243 (2000).

${ }^{8}$ M. Giovannini, Ph.D. thesis, No. 2169, Ecole Polytechnique Fédérale de Lausanne (2000). 
${ }^{9}$ H. S. Bergh, B. Gergen, H. Nienhaus, A. Majumdar, W. H. Weinberg, and E. W. McFarland, Rev. Sci. Instrum. 70, 2087 (1999).

${ }^{10}$ J.-W. Lee, J.-R. Jeong, D.-H. Kim, J. S. Ahn, J. Kim, and S.-C. Shin, Rev. Sci. Instrum. 71, 3801 (2000).

${ }^{11}$ S. Boukari, J. Balay, E. Beaurepaire, G. Biechel, B. Carriere, J. P. Deville, B. Muller, and F. Scheurer, Vacuum 52, 327 (1999).

${ }^{12}$ N. Weiss, Ph.D. thesis, No. 2980, Ecole Polytechnique Fédérale de Lausanne (2004).

${ }^{13}$ J. Zak, E. R. Moog, C. Liu, and S. D. Bader, Phys. Rev. B 43, 6423 (1991).

${ }^{14}$ A. Vernes, I. Reichl, P. Weinberger, L. Szunyogh, and C. Sommers, Phys. Rev. B 70, 195407 (2004).

${ }^{15}$ G. Metzger, P. Pluvinage, and R. Torguet, Ann. Phys. 10, 5 (1965).

${ }^{16}$ P. B. Johnson and R. W. Christy, Phys. Rev. B 9, 5056 (1974).

${ }^{17}$ L. Schulz, J. Opt. Soc. Am. 44, 357 (1954).

${ }^{18}$ L. Schulz and F. R. Tangherlini, J. Opt. Soc. Am. 44, 362 (1954).

${ }^{19}$ J. H. Weaver, C. G. Olson, and D. W. Lynch, Phys. Rev. B 15, 4115 (1977).
${ }^{20}$ R. Carey and B. W. J. Thomas, J. Phys. D 7, 2362 (1974).

${ }^{21}$ G. Baudot, S. Rohart, V. Repain, H. Ellmer, Y. Girard, and S. Rousset, Appl. Surf. Sci. 212, 360 (2003).

${ }^{22}$ S. Rohart, V. Repain, A. Tejeda, P. Ohresser, F. Scheurer, P. Bencok, J. Ferre, and S. Rousset, Phys. Rev. B 73, 165412 (2006).

${ }^{23}$ R. Skomski, H.-P. Oepen, and J. Kirschner, Phys. Rev. B 58, 3223 (1998).

${ }^{24}$ A. Vaterlaus, O. Portmann, C. Stamm, and D. Pescia, J. Magn. Magn. Mater. 272, 1137 (2004).

${ }^{25}$ J. V. Barth, H. Brune, G. Ertl, and R. J. Behm, Phys. Rev. B 42, 9307 (1990).

${ }^{26}$ T. Koide, H. Miyauchi, J. Okamoto, T. Shidara, A. Fujimori, H. Fukutani, K. Amemiya, H. Takeshita, S. Yuasa, T. Katayama, and Y. Suzuki, Phys. Rev. Lett. 87, 257201 (2001).

${ }^{27}$ J. J. Lu, H. L. Huang, and I. Klik, J. Appl. Phys. 76, 1726 (1994).

${ }^{28}$ H. A. Dürr, S. S. Dhesi, E. Dudzik, D. Knabben, G. van der Laan, J. B. Goedkoop, and F. U. Hillebrecht, Phys. Rev. B 59, R701 (1999).

${ }^{29}$ R. W. Chantrell, N. Walmsley, J. Gore, and M. Maylin, Phys. Rev. B 63, 024410 (2000). 